\title{
Enumeration and asymptotic properties of unlabeled outerplanar graphs
}

\author{
Manuel Bodirsky ${ }^{1}$, Éric Fusy ${ }^{2}$, Mihyun Kang ${ }^{1,3}$, and Stefan Vigerske ${ }^{1}$ \\ ${ }^{1}$ Humboldt-Universität zu Berlin, Institut für Informatik \\ Unter den Linden 6, 10099 Berlin, Germany \\ \{bodirsky, kang\}@informatik.hu-berlin.de \\ stefan@mathematik.hu-berlin.de \\ ${ }^{2}$ Projet Algo, INRIA Rocquencourt \\ B. P. 105, 78153 Le Chesnay Cedex, France \\ eric.fusy@inria.fr
}

Submitted: Mar 15, 2007; Accepted: Aug 1, 2007; Published: Sep 14, 2007

Mathematics Subject Classification: $05 \mathrm{C} 88$

\begin{abstract}
We determine the exact and asymptotic number of unlabeled outerplanar graphs. The exact number $g_{n}$ of unlabeled outerplanar graphs on $n$ vertices can be computed in polynomial time, and $g_{n}$ is asymptotically $g n^{-5 / 2} \rho^{-n}$, where $g \approx 0.00909941$ and $\rho^{-1} \approx 7.50360$ can be approximated. Using our enumerative results we investigate several statistical properties of random unlabeled outerplanar graphs on $n$ vertices, for instance concerning connectedness, the chromatic number, and the number of edges. To obtain the results we combine classical cycle index enumeration with recent results from analytic combinatorics.

Keywords: unlabeled outerplanar graphs, dissections, combinatorial enumeration, cycle index, asymptotic estimates, singularity analysis
\end{abstract}

\section{Introduction and results}

Singularity analysis is very successful for the asymptotic enumeration of combinatorial structures [14], once a sufficiently good description of the corresponding generating functions is provided. When we count unlabeled structures, i.e., when we count the structures

\footnotetext{
${ }^{3}$ Research supported by the Deutsche Forschungsgemeinschaft (DFG Pr 296)
} 
up to isomorphism, the potential symmetries of the structures often require a more powerful tool than generating functions, e.g., cycle index sums, introduced by Pólya [30]. From the cycle index sums for classes of combinatorial structures we can obtain the corresponding generating functions, to which we can then apply singularity analysis. However, when the cycle index sums are given only implicitly, it might be a challenging task to apply this technique. This is well illustrated by the attempts for the enumeration of planar graphs: The asymptotic number of labeled planar graphs was recently determined by Giménez and Noy [20], based on singularity analysis, whereas the enumeration of unlabeled planar graphs has been left open for several decades [39].

In this paper we determine the exact and asymptotic number of unlabeled outerplanar graphs, an important subclass of the class of all unlabeled planar graphs. We provide a polynomial-time algorithm to compute the exact number $g_{n}$ of unlabeled outerplanar graphs on $n$ vertices, and prove that $g_{n}$ is asymptotically $g n^{-5 / 2} \rho^{-n}$, where $g \approx 0.00909941$ and $\rho^{-1} \approx 7.50360$ can be approximated. Building on our enumerative results we derive typical properties of a random unlabeled outerplanar graph on $n$ vertices (i.e., a graph chosen uniformly at random among all unlabeled outerplanar graphs on $n$ vertices), for example connectedness, the chromatic number, the number of components, and the number of edges (see Section 5).

Before we provide a more detailed exposition of the main results of this paper, we give a brief survey on the vast literature on enumerative results for planar structures. The exact and asymptotic number of embedded planar graphs (i.e., planar maps) has been studied intensively, starting with Tutte's seminal work on the number of rooted oriented planar maps [35]. The number of three-connected planar maps is related to the number of three-connected planar graphs [25, 35], since a three-connected planar graph has a unique embedding on the sphere [40]. Bender, Gao, and Wormald used this property to count labeled two-connected planar graphs [2], and Giménez and Noy extended this work to the enumeration of labeled planar graphs [20]. Many interesting properties of a random labeled planar graph were studied in $[11,18,19,24,27]$. It is also known how to generate labeled three-connected planar graphs, labeled planar maps, and labeled planar graphs uniformly at random $[5,8,16,17,33]$.

The asymptotic number of general unlabeled planar graphs has not yet been determined, but has been studied for quite some time [39]. Moreover, no polynomial time algorithm for the computation of the exact number of unlabeled planar graphs on $n$ vertices is known. Such an algorithm is only known for unlabeled rooted two-connected planar graphs [6], and for unlabeled rooted cubic planar graphs [7].

An outerplanar graph is a graph that can be embedded in the plane such that every vertex is incident to the outer face. Such graphs can also be characterized in terms of forbidden minors [10], namely $K_{2,3}$ and $K_{4}$. The class of outerplanar graphs is often used as a first non-trivial test-case for results about the class of all planar graphs; apart from that, this class appears frequently in various applications of graph theory. Two-connected outerplanar graphs can be identified with dissections of a convex polygon (see, e.g., [4]). Further, Read provided counting formulas for the number of unlabeled two-connected outerplanar graphs [31]. General outerplanar graphs can be decomposed according to their 
degree of connectivity: an outerplanar graph is a set of connected outerplanar graphs, and a connected outerplanar graph can be decomposed into two-connected blocks.

In the labeled case this decomposition yields equations that link the exponential generating functions of two-connected, connected, and general outerplanar graphs. Once labeled dissections are enumerated, these equations yield formulas for counting outerplanar graphs. The asymptotic number of labeled outerplanar graphs was recently determined [4].

In the unlabeled case the same decomposition can be used, but generating functions have to be replaced by cycle index sums (as introduced by Pólya [30]) to deal with potential symmetries [21, page 188]. From cycle index sums we obtain implicit equations for the ordinary generating functions for unlabeled outerplanar graphs (see Section 3). We then apply singularity analysis, a very powerful tool that is thoroughly developed in the forthcoming book of Flajolet and Sedgewick [14]. A similar strategy was applied by Labelle, Lamathe, and Leroux for the enumeration of unlabeled $k$-gonal 2 -trees [22]. However, the singularity analysis for outerplanar graphs is more challenging. A new difficulty we have to face is that the generating function for connected outerplanar graphs is defined implicitly via substitution into 2-connected components. Consequently, finding the singular development for this series requires a careful treatment of cases when applying the singular implicit function theorem (see Section 4.1 for the details). Singular developments then make it possible to obtain the asymptotic results.

Contributions. From now on we always consider outerplanar graphs as unlabeled objects, unless stated otherwise. Our first result is the exact and asymptotic number of unlabeled outerplanar graphs.

Theorem 1.1. The exact numbers of two-connected outerplanar graphs $d_{n}$, connected outerplanar graphs $c_{n}$, and outerplanar graphs $g_{n}$ with $n$ vertices can be computed in polynomial time.

See the sequences A001004, A111563, and A111564 from [34] for initial values.

Theorem 1.2. The numbers $d_{n}, c_{n}$, and $g_{n}$ of two-connected, connected, and general outerplanar graphs with $n$ vertices have the asymptotic estimates

$$
\begin{aligned}
& d_{n} \sim d n^{-5 / 2} \delta^{-n}, \\
& c_{n} \sim c n^{-5 / 2} \rho^{-n}, \\
& g_{n} \sim g n^{-5 / 2} \rho^{-n},
\end{aligned}
$$

with exponential growth rates $\delta^{-1}=3+2 \sqrt{2} \approx 5.82843$ and $\rho^{-1} \approx 7.50360$, and constants $d \approx 0.00596026, c \approx 0.00760471$, and $g \approx 0.00909941$. (See Theorems 4.1, 4.3, and 4.4.)

The growth rates for the labeled case are given in [4, 13]. Observe that the exponential growth rates of unlabeled and labeled two-connected outerplanar graphs coincide. Hence, asymptotically almost all two-connected outerplanar graphs are asymmetric. For the connected and general case, the growth rates differ. 
Having the asymptotic estimates of connected outerplanar graphs and outerplanar graphs, we investigate asymptotic distributions of parameters such as the number of components and the number of isolated vertices of a random outerplanar graph (i.e., a graph chosen uniformly at random among all outerplanar graphs on $n$ vertices) as $n$ tends to infinity.

Theorem 1.3. (1) The probability that a random outerplanar graph is connected is asymptotically $c / g \approx 0.845721$.

(2) The expected number of components in a random outerplanar graph is asymptotically equal to a constant $\approx 1.17847$.

(3) The asymptotic distribution of the number of isolated vertices in a random outerplanar graph follows a geometric law with parameter $\rho$. In particular, the expected number of isolated vertices in a random outerplanar graph is asymptotically $\rho /(1-\rho) \approx 0.153761$.

Next, we study the distribution of the number of edges in a random outerplanar graph.

Theorem 1.4. The distribution of the number of edges in a random outerplanar graph on $n$ vertices is asymptotically Gaussian with mean $\mu$ and variance $\sigma^{2} n$, where $\mu \approx 1.54894$ and $\sigma^{2} \approx 0.227504$. The same holds for a random connected outerplanar graph with the same mean and variance and for a random two-connected outerplanar graph with asymptotic mean $(1+\sqrt{2} / 2) n \approx 1.70711 n$ and asymptotic variance $\sqrt{2} / 8 n \approx 0.176777 n$.

Further, we study the chromatic number of a random outerplanar graph. An outerplanar graph is easily shown to be 3-colourable. In order to further investigate the distribution of the chromatic number of a random outerplanar graph we also estimate the asymptotic number of bipartite outerplanar graphs.

Theorem 1.5. The number of bipartite outerplanar graphs $\left(g_{b}\right)_{n}$ on $n$ vertices has the asymptotic estimate $\left(g_{b}\right)_{n} \sim b n^{-5 / 2} \rho_{b}^{-n}$, with $\rho_{b}^{-1} \approx 4.57717$.

The fact that the growth constant of bipartite outerplanar graphs is smaller than the growth constant of outerplanar graphs yields the following result:

Theorem 1.6. The probability that the chromatic number of a random outerplanar graph is different from three converges to zero exponentially fast.

\section{Preliminaries}

We recall some concepts and techniques that we need for the enumeration of unlabeled graphs, and some facts from singularity analysis to obtain asymptotic estimates. 


\subsection{Cycle index sums and ordinary generating functions}

To enumerate unlabeled graphs, cycle index sums were introduced by Pólya (see e.g., [21, 30]). For a group of permutations $A$ on an object set $X=\{1, \ldots, n\}$ (for example, the vertex set of a graph), the cycle index $Z(A)$ of $A$ with respect to the formal variables $s_{1}, \ldots, s_{n}$ is defined by

$$
Z(A):=Z\left(A ; s_{1}, s_{2}, \ldots\right):=\frac{1}{|A|} \sum_{\alpha \in A} \prod_{k=1}^{n} s_{k}^{j_{k}(\alpha)},
$$

where $j_{k}(\alpha)$ denotes the number of cycles of length $k$ in the decomposition of $\alpha \in A$ into disjoint cycles. For a graph $G$ on $n$ vertices with automorphism group $\Gamma(G)$, we write $Z(G):=Z(\Gamma(G))$, and for a set of graphs $\mathcal{K}$, we write $Z(\mathcal{K})$ for the cycle index sum for $\mathcal{K}$ defined by

$$
Z(\mathcal{K}):=Z\left(\mathcal{K} ; s_{1}, s_{2}, \ldots\right):=\sum_{K \in \mathcal{K}} Z\left(K ; s_{1}, s_{2}, \ldots\right) .
$$

It can be shown [3] that, if $\overline{\mathcal{K}}$ is the corresponding class of labeled graphs, then

$$
Z(\mathcal{K})=\sum_{n \geq 0} \frac{1}{n !} \sum_{K \in \overline{\mathcal{K}}_{n}} \sum_{\alpha \in \Gamma(K)} \prod_{k=1}^{n} s_{k}^{j_{k}(\alpha)}
$$

which coincides with the classical definition of a cycle index series and shows the close relationship of cycle index sums to exponential generating functions in labeled counting.

Indeed, cycle index sums can be used for the enumeration of unlabeled structures in a similar way as generating functions for labeled enumeration. First of all, the composition of graphs corresponds to the composition of the associated cycle indices. Consider an object set $X=\{1, \ldots, n\}$ and a permutation group $A$ on $X$. A composition of $n$ graphs from $\mathcal{K}$ is a function $f: X \rightarrow \mathcal{K}$. Two compositions $f$ and $g$ are similar, $f \sim g$, if there exists a permutation $\alpha \in A$ with $f \circ \alpha=g$. We write $\mathcal{G}$ for the set of equivalence classes of compositions of $n$ graphs from $\mathcal{K}$ (with respect to the equivalence relation $\sim$ ). Then

$$
Z(\mathcal{G})=Z(A)[Z(\mathcal{K})]:=Z\left(A ; Z\left(\mathcal{K} ; s_{1}, s_{2}, \ldots\right), Z\left(\mathcal{K} ; s_{2}, s_{4}, \ldots\right), \ldots\right),
$$

i.e., $Z(\mathcal{G})$ is obtained from $Z(A)$ by replacing each $s_{i}$ by $Z\left(\mathcal{K} ; s_{i}, s_{2 i}, \ldots\right)$ [21]. Hence, Formula (2.1) makes it possible to derive the cycle index sum for a class of graphs by decomposing the graphs into simpler structures with a known cycle index sum.

In many cases, such a decomposition is only possible when, for example, one vertex is distinguished from the others in the graphs, so that there is a unique point where the decomposition is applied. Graphs with a distinguished vertex are called vertex rooted graphs. The automorphism group of a vertex rooted graph consists of all automorphisms of the unrooted graph that fix the root vertex. Hence, one can expect a close relation between the cycle index sum for unrooted graphs and the cycle index sum for their rooted counterparts. As shown in [21], if $\mathcal{G}$ is an unlabeled set of graphs and $\hat{\mathcal{G}}$ is the set of graphs of $\mathcal{G}$ rooted at a vertex, then

$$
Z(\hat{\mathcal{G}})=s_{1} \frac{\partial}{\partial s_{1}} Z(\mathcal{G})
$$


This relationship can be inverted to express the cycle index sum for the unrooted graphs in terms of the cycle index sum for the rooted graphs,

$$
Z(\mathcal{G})=\left.\int_{0}^{s_{1}} \frac{1}{t_{1}} Z(\hat{\mathcal{G}})\right|_{s_{1}=t_{1}} d t_{1}+\left.Z(\mathcal{G})\right|_{s_{1}=0}
$$

Observe that permutations without fixed points are not counted by the cycle indices of the rooted graphs, so that their cycle indices are added as a boundary term to $Z(\mathcal{G})$.

Once the cycle index sum for a class of graphs of interest is known, the corresponding ordinary generating function can be derived by replacing the formal variables $s_{i}$ in the cycle index sums by $x^{i}$ (note that $Z\left(G ; x, x^{2}, \ldots\right)=x^{|G|}$ for a graph $G$, where $|G|$ denotes the number of vertices of $G$ ). More generally, for a group $A$ and an ordinary generating function $K(x)$ we define

$$
Z(A ; K(x)):=Z\left(A ; K(x), K\left(x^{2}\right), K\left(x^{3}\right), \ldots\right)
$$

as the ordinary generating function obtained by substituting each $s_{i}$ in $Z(A)$ by $K\left(x^{i}\right)$, $i \geq 1$.

\subsection{Singularity analysis}

To determine asymptotic estimates of the coefficients of a generating function we use singularity analysis [14]. The fundamental observation is that the exponential growth of the coefficients of a complex-valued function that is analytic at the origin is determined by the dominant singularities of the function, i.e., singularities at the boundary of the disc of convergence. By Pringsheim's theorem [14, Thm. IV.6], a generating function $F(x)$ with non-negative coefficients and finite radius of convergence $R$ has a singularity at the point $x=R$. If $x=R$ is the unique singularity on the disk $|z|=R$, it follows from the exponential growth formula [14, Thm. IV.7] that the coefficients $f_{n}=\left[x^{n}\right] F(x)$ satisfy $f_{n}=\theta(n) R^{-n}$ with $\limsup _{n \rightarrow \infty}|\theta(n)|^{1 / n}=1$. A closer look at the type of the dominant singularity, for example, the order of the pole, enables the computation of subexponential factors as well. The following lemma describes the singular expansion for a common case [14, Thm. VI.1].

Lemma 2.1 (standard function scale). Let $F(x)=(1-x)^{-\alpha}$ with $\alpha \notin\{0,-1,-2, \ldots\}$. Then the coefficients $f_{n}$ of $F(x)$ have a full asymptotic development in descending powers of $n$,

$$
f_{n}=\left(\begin{array}{c}
n+\alpha-1 \\
n
\end{array}\right) \sim \frac{n^{\alpha-1}}{\Gamma(\alpha)}\left(1+\sum_{k=1}^{\infty} \frac{e_{k}(\alpha)}{n^{k}}\right)
$$

where $\Gamma(\alpha)$ is the Gamma-Function, $\Gamma(\alpha):=\int_{0}^{\infty} e^{-t} t^{\alpha-1} d t$ for $\alpha \notin\{0,-1,-2, \ldots\}$, and $e_{k}(\alpha)$ is a polynomial in $\alpha$ of degree $2 k$.

In our calculations, it will appear that a generating function $F(x)$ is given only implicitly by an equation $H(x, F(x))=0$. Theorem VII.3 in [14] describes how to derive 
a full singular expansion of $F(x)$ in this case. We state it here in a slightly modified version. A generating function is called aperiodic, if it can not be written in the form $Y(x)=x^{a} \tilde{Y}\left(x^{d}\right)$ with $d \geq 2$ and $\tilde{Y}(x)$ analytic at 0 .

Theorem 2.2 (singular implicit functions). Let $H(x, y)$ be a bivariate function that is analytic in a complex domain $|x|<R,|y|<S$ and verifies $H(0,0)=0, \frac{\partial}{\partial y} H(0,0)=-1$, and whose Taylor coefficients $h_{m, n}$ satisfy the following positivity conditions: they are nonnegative except for $h_{0,1}=-1$ (because $\frac{\partial}{\partial y} H(0,0)=-1$ ) and $h_{m, n}>0$ for at least one pair $(m, n)$ with $n \geq 2$. Assume that there are two numbers $\rho \in(0, R)$ and $\tau \in(0, S)$ such that

$$
H(\rho, \tau)=0, \quad \frac{\partial}{\partial y} H(\rho, \tau)=0, \quad \frac{\partial^{2}}{\partial y^{2}} H(\rho, \tau) \neq 0, \quad \text { and } \quad \frac{\partial}{\partial x} H(\rho, \tau) \neq 0 .
$$

Assume further that the equation $H(x, Y(x))=0$ admits a solution $Y(x)$ that is analytic at 0 , has non-negative coefficients, and is aperiodic. Then $\rho$ is the unique dominant singularity of $Y(x)$, and $Y(x)$ converges at $x=\rho$, where it has the singular expansion

$$
Y(x)=\tau+\sum_{i \geq 1} Y_{i}\left(\sqrt{1-\frac{x}{\rho}}\right)^{i}, \quad \text { with } Y_{1}=-\sqrt{\frac{2 \rho \frac{\partial}{\partial x} H(\rho, \tau)}{\frac{\partial^{2}}{\partial y^{2}} H(\rho, \tau)}} \neq 0,
$$

and computable constants $Y_{2}, Y_{3}, \cdots$. Hence,

$$
\left[x^{n}\right] Y(x)=-\frac{Y_{1}}{2 \sqrt{\pi n^{3}}} \rho^{-n}\left(1+O\left(\frac{1}{n}\right)\right) .
$$

The first two equations in (2.5) are condition $\mathrm{I}_{3}$ in [14, Def. II.4], whereas the latter two equations are to ensure that $Y_{1}$ is well-defined and nonzero. The formulas that express the coefficients $Y_{i}$ in terms of partial derivatives of $H(x, y)$ at $(\rho, \tau)$ can be found in $[12,29]$.

When a parameter $\xi$ of a combinatorial structure is studied, the generating function $F(x)$ has to be extended to a bivariate generating function $F(x, y)=\sum_{n, m} f_{n, m} x^{n} y^{m}$ where the second variable $y$ marks $\xi$. We can determine the asymptotic distribution of $\xi$ from $F(x, y)$ by varying $y$ in some neighbourhood of 1 . The following theorem follows from the so-called quasi-powers theorem [14, Thm. IX.7].

Theorem 2.3. Let $F(x, y)$ be a bivariate generating function of a family of objects $\mathcal{F}$, where the power in $y$ corresponds to a parameter $\xi$ on $\mathcal{F}$, i.e., $\left[x^{n} y^{m}\right] F(x, y)=\mid\{F \in$ $\mathcal{F}|| F \mid=n, \xi(F)=m\} \mid$. Assume that, in a fixed complex neighbourhood of $y=1, F(x, y)$ has a singular expansion of the form

$$
F(x, y)=\sum_{k \geq 0} F_{k}(y)\left(\sqrt{1-\frac{x}{\rho(y)}}\right)^{k}
$$

where $\rho(y)$ is the dominant singularity of $x \mapsto F(x, y)$. Furthermore, assume that there is an odd $k_{0} \in \mathbb{N}$ such that for all $y$ in the neighbourhood of $1, F_{k_{0}}(y) \neq 0$ and $F_{k}(y)=0$ 
for $0<k<k_{0}$ odd. Assume that $\rho(y)$ and $F_{k_{0}}(y)$ are analytic at $y=1$, and that $\rho(y)$ satisfies the variance condition, $\rho^{\prime \prime}(1) \rho(1)+\rho^{\prime}(1) \rho(1)-\rho^{\prime}(1)^{2} \neq 0$.

Let $X_{n}$ be the restriction of $\xi$ onto all objects in $\mathcal{F}$ of size $n$. Under these conditions, the distribution of $X_{n}$ is asymptotically Gaussian with mean

$$
\mathbb{E}\left[X_{n}\right] \sim \mu n \quad \text { with } \quad \mu=-\frac{\rho^{\prime}(1)}{\rho(1)}
$$

and variance

$$
\mathbb{V}\left[X_{n}\right] \sim \sigma^{2} n \quad \text { with } \quad \sigma^{2}=-\frac{\rho^{\prime \prime}(1)}{\rho(1)}-\frac{\rho^{\prime}(1)}{\rho(1)}+\left(\frac{\rho^{\prime}(1)}{\rho(1)}\right)^{2} .
$$

The speed of convergence is $O\left(n^{-1 / 2}\right)$, that is

$$
\mathbb{P}\left[\frac{X_{n}-\mu n}{\sigma_{n} \sqrt{n}} \leq x\right]=\Phi(x)+O\left(n^{-1 / 2}\right),
$$

where $\Phi(x)$ denotes the cumulative distribution function of the standard normal distribution.

\section{$3 \quad$ Exact enumeration of outerplanar graphs}

In the next sections we derive the cycle index sums for rooted and unrooted two-connected, rooted and unrooted connected, general, and bipartite outerplanar graphs.

\subsection{Enumeration of dissections (two-connected outerplanar graphs)}

A graph is two-connected if at least two of its vertices have to be removed to disconnect it. It is well known that a two-connected outerplanar graph with at least three vertices has a unique Hamiltonian cycle and can therefore be embedded uniquely in the plane so that this Hamiltonian cycle is the contour of the outer face. This unique embedding is thus a dissection of a convex polygon. Hence, the task of counting two-connected outerplanar graphs coincides with the task of counting dissections of a polygon. Furthermore, changing to the dual of a dissection, it is seen that the task of counting dissections coincides with the task of counting embedded trees with no vertex of degree 2. Read utilized this property to derive the generating function for unlabeled dissections [31]. First, he derived the generating functions for several types of vertex, edge, and face rooted dissections, then he used these functions to express the generating function for unrooted dissections by an application of the dissimilarity characteristic theorem for trees [21, page 56]. Vigerske [37] extended Read's work to derive the following cycle index sums. We denote the set of two-connected outerplanar graphs (i.e., dissections) by $\mathcal{D}$ and the set of vertex rooted two-connected outerplanar graphs by $\mathcal{V}$. 
Theorem 3.1. The cycle index sum for two-connected outerplanar graphs is given by

$$
\begin{aligned}
Z(\mathcal{D})= & -\frac{1}{2} \sum_{d \geq 1} \frac{\varphi(d)}{d} \log \left(\frac{3}{4}-\frac{1}{4} s_{d}+\frac{1}{4} \sqrt{s_{d}^{2}-6 s_{d}+1}\right)+\frac{s_{2}+s_{1}^{2}-4 s_{1}-2}{16} \\
& +\frac{s_{1}^{2}-3 s_{1}^{2} s_{2}+2 s_{1} s_{2}}{16 s_{2}^{2}}+\frac{3-s_{1}}{16} \sqrt{s_{1}^{2}-6 s_{1}+1}-\frac{1}{16}\left(1+\frac{s_{1}^{2}}{s_{2}^{2}}+\frac{2 s_{1}}{s_{2}}\right) \sqrt{s_{2}^{2}-6 s_{2}+1}
\end{aligned}
$$

where $\varphi$ denotes the Euler- $\varphi$-function, defined as follows: $\varphi(n)=n \prod_{p \mid n}\left(1-p^{-1}\right), n \in \mathbb{N}$, where the product is over all prime numbers $p$ which divide $n$.

Using Formula (2.2) and Theorem 3.1 we derive the cycle index sum for vertex rooted dissections, which we will need later.

Corollary 3.2. The cycle index sum for vertex rooted dissections is given by

$$
Z\left(\mathcal{V} ; s_{1}, s_{2}\right)=\frac{s_{1}}{8}\left(1+s_{1}-\sqrt{s_{1}^{2}-6 s_{1}+1}\right)+\frac{s_{1}}{8 s_{2}^{2}}\left(s_{1}+s_{2}\right)\left(1-3 s_{2}-\sqrt{s_{2}^{2}-6 s_{2}+1}\right) .
$$

\subsection{Enumeration of connected outerplanar graphs}

We denote the set of unrooted connected outerplanar graphs by $\mathcal{C}$, and the set of vertex rooted connected outerplanar graphs by $\hat{\mathcal{C}}$. All rooted graphs considered in this section are rooted at a vertex. Again, ordinary generating functions are denoted by capital letters and coefficients by small letters. Thus, $\hat{C}(x)=\sum_{n} \hat{c}_{n} x^{n}$ and $C(x)=\sum_{n} c_{n} x^{n}$.

The cycle index sum for rooted connected outerplanar graphs is derived by decomposing the graphs into rooted two-connected outerplanar graphs, i.e., vertex rooted dissections. First, every connected outerplanar graph rooted at a cut-vertex is decomposed into a set of non-cut-vertex rooted connected outerplanar graphs. Then, a non-cut-vertex rooted connected outerplanar graph can be constructed unambiguously by taking a rooted dissection and attaching a rooted connected outerplanar graph at each vertex of the dissection other than the root vertex. This decomposition goes back to Norman [26] and was generalized by Robinson [32] and Harary and Palmer [21, page 188] for general graphs.

Lemma 3.3 (rooted connected outerplanar graphs). The cycle index sum for vertex rooted connected outerplanar graphs is implicitly determined by the equation

$$
Z(\hat{\mathcal{C}})=s_{1} \exp \left(\sum_{k \geq 1} \frac{Z\left(\mathcal{V} ; Z\left(\hat{\mathcal{C}} ; s_{k}, s_{2 k}, \ldots\right), Z\left(\hat{\mathcal{C}} ; s_{2 k}, s_{4 k}, \ldots\right)\right)}{k Z\left(\hat{\mathcal{C}} ; s_{k}, s_{2 k}, \ldots\right)}\right) .
$$

To derive the cycle index sum for unrooted connected outerplanar graphs, one can use Formula (2.3). The boundary term in (2.3) corresponds here to connected outerplanar graphs with no fixed vertex. Since each fixed-point free permutation in a connected graph has a unique block whose vertices are setwise fixed by the automorphisms of the graph [21, page 190], this term can be replaced by $\left.Z(\mathcal{D})\right|_{s_{1}=0}[Z(\hat{\mathcal{C}})]$. Using the special structure $(3.3)$ of $Z(\hat{\mathcal{C}})$, a closed solution for the integral in $(2.3)$ can be found $[32,37]$ and we obtain the following result. 
Theorem 3.4 (connected outerplanar graphs). The cycle index sum for connected outerplanar graphs is given by

$$
Z(\mathcal{C})=Z(\hat{\mathcal{C}})+Z(\mathcal{D} ; Z(\hat{\mathcal{C}}))-Z(\mathcal{V} ; Z(\hat{\mathcal{C}}))
$$

Replacing $s_{i}$ by $x^{i}$ in $Z(\hat{\mathcal{C}})$, the generating function $\hat{C}(x)$ counting vertex rooted connected outerplanar graphs satisfies

$$
\hat{C}(x)=x \exp \left(\sum_{k \geq 1} \frac{Z\left(\mathcal{V} ; \hat{C}\left(x^{k}\right), \hat{C}\left(x^{2 k}\right)\right)}{k \hat{C}\left(x^{k}\right)}\right),
$$

from which the coefficients $\hat{C}_{n}$ counting vertex rooted connected outerplanar graphs can be extracted in polynomial time: $\hat{C}(x)=x+x^{2}+3 x^{3}+10 x^{4}+40 x^{5}+181 x^{6}+918 x^{7}+\ldots$, see $[36,37]$ for more entries. The numbers in [36] verify the correctness of our result and were computed by the polynomial algorithm proposed in [9].

In addition, it follows from (3.4) that the generating function $C(x)$ counting connected outerplanar graphs satisfies

$$
C(x)=\hat{C}(x)+Z(\mathcal{D} ; \hat{C}(x))-Z(\mathcal{V} ; \hat{C}(x)),
$$

from which the coefficients $c_{n}$ can be extracted in polynomial time: $C(x)=x+x^{2}+2 x^{3}+$ $5 x^{4}+13 x^{5}+46 x^{6}+172 x^{7}+\ldots$, see [34, A111563] for more entries.

\subsection{Enumeration of outerplanar graphs}

We denote the set of outerplanar graphs by $\mathcal{G}$, its ordinary generating function by $G(x)$ and the number of outerplanar graphs with $n$ vertices by $g_{n}$. As an outerplanar graph is a collection of connected outerplanar graphs, it is now easy to obtain the cycle index sum for outerplanar graphs. An application of the composition formula (2.1) with the symmetric group $S_{l}$ and object set $\mathcal{C}$ yields that $Z\left(S_{l}\right)[Z(\mathcal{C})]$ is the cycle index sum for outerplanar graphs with $l$ connected components. Thus, by summation over all $l \geq 0$ (we include also the empty graph into $\mathcal{G}$ for convenience), we obtain the following theorem.

Theorem 3.5 (outerplanar graphs). The cycle index sum for outerplanar graphs is given by

$$
Z(\mathcal{G})=\exp \left(\sum_{k \geq 1} \frac{1}{k} Z\left(\mathcal{C} ; s_{k}, s_{2 k}, \ldots\right)\right) .
$$

Hence the generating functions $G(x)$ and $C(x)$ of outerplanar and connected outerplanar graphs are related by

$$
G(x)=\exp \left(\sum_{k \geq 1} \frac{1}{k} C\left(x^{k}\right)\right) .
$$

From this, we can extract in polynomial time the coefficients $g_{n}$ counting outerplanar graphs, $G(x)=1+x+2 x^{2}+4 x^{3}+10 x^{4}+25 x^{5}+80 x^{6}+277 x^{7}+\ldots$, see [34, A111564] for more entries. 


\subsection{Enumeration of bipartite outerplanar graphs}

To study the chromatic number of a typical outerplanar graph we enumerate bipartite outerplanar graphs. Observe that an outerplanar graph is bipartite if and only if all of its blocks are bipartite. As discussed, blocks of an outerplanar graph are dissections, and it is clear that a dissection is bipartite when all of its inner faces have an even number of vertices. The decomposition for (general) dissections can be adapted to dissections where all faces have even degree. Once the cycle index sum for bipartite dissections is obtained, the computation of the cycle index sums for bipartite connected outerplanar graphs, and then for bipartite outerplanar graphs works in the same way as for the general case, see [37] for details. From that the coefficients of the series $G_{b}(x)$ counting bipartite outerplanar graphs can be extracted in polynomial time: $G_{b}(x)=1+x+x^{2}+x^{3}+7 x^{4}+$ $12 x^{5}+29 x^{6}+61 x^{7}+\ldots$, see the sequences A111757, A111758, and A111759 of [34] for the coefficients of two-connected, connected, and general bipartite outerplanar graphs.

\section{Asymptotic enumeration of unlabeled outerplanar graphs}

To determine the asymptotic number of two-connected, connected, and general outerplanar graphs, we use singularity analysis as introduced in Section 2.2. To compute the growth constants and subexponential factors we expand the generating functions for outerplanar graphs around their dominant singularities. For unlabeled two-connected outerplanar graphs we present an analytic expression of the growth constant. For the connected and the general case we give numerical approximations of the growth constants in Section 4.2.

\subsection{Asymptotic estimates}

We now prove the first part of Theorem 1.2 on the asymptotic number of dissections.

Theorem 4.1 (asymptotic number of unrooted dissections). The number $d_{n}$ of unlabeled two-connected outerplanar graphs on $n$ vertices has the asymptotic estimate $d_{n} \sim d n^{-5 / 2} \delta^{-n}$ with growth rate $\delta^{-1}=3+2 \sqrt{2} \approx 5.82843$ and constant $d \approx 0.00596026$.

Proof. The smallest root of $x^{2}-6 x+1$ is $\delta=3-2 \sqrt{2}$. Equation (3.1) implies that $D(x)=Z\left(\mathcal{D} ; x, x^{2}, \ldots\right)$ can be written as

$$
D(x)=-\frac{1}{2} \log \left(1-\frac{\sqrt{x^{2}-6 x+1}}{x-3}\right)+\frac{3-x}{16} \sqrt{x^{2}-6 x+1}+A(x),
$$

where $A(x)$ is analytic at 0 with radius of convergence $>\delta$. Since the logarithmic term is analytic for $|x|<\delta$, we can expand it and collect ascending powers of $\sqrt{x^{2}-6 x+1}$ in $D(x)$. Thus,

$$
D(x)=\left(-\frac{1}{16(x-3)}+\frac{1}{6(x-3)^{3}}\right)\left(\sqrt{x^{2}-6 x+1}\right)^{3}+\sum_{k \geq 4} \frac{1}{2 k}\left(\frac{\sqrt{x^{2}-6 x+1}}{x-3}\right)^{k}+\tilde{A}(x),
$$


where $\tilde{A}(x)$ is again analytic at 0 with radius of convergence $>\delta$.

Finally, using $\sqrt{x^{2}-6 x+1}=\sqrt{1-x / \delta} \sqrt{1-\delta x}$ for $x \leq \delta$ and applying Lemma 2.1 we obtain

$$
\begin{aligned}
d_{n} & =\left(-\frac{1}{16(\delta-3)}+\frac{1}{6(\delta-3)^{3}}\right)\left(\sqrt{1-\delta^{2}}\right)^{3} \frac{1}{\Gamma(-3 / 2)} n^{-5 / 2} \delta^{-n}\left(1+O\left(\frac{1}{n}\right)\right) \\
& \sim \frac{\sqrt{99 \sqrt{2}-140}}{8 \sqrt{\pi}} n^{-5 / 2}(3+2 \sqrt{2})^{n} .
\end{aligned}
$$

For the number of dissections with $n$ vertices that are labeled clockwise Flajolet and Noy in [13] determined the asymptotic estimate $\frac{1}{4 \sqrt{\pi}} \sqrt{99 \sqrt{2}-140} n^{-3 / 2}(3+2 \sqrt{2})^{n}$.

We now turn to the problem of asymptotic enumeration of connected outerplanar graphs. First we have to establish the singular development of the generating function for vertex rooted connected outerplanar graphs $\hat{C}(x)$. Let $\rho$ be the radius of convergence of $\hat{C}(x)$. Observe that the coefficients $\hat{c}_{n}$ are bounded from below by the number of unlabeled vertex rooted dissections $v_{n}$, which have exponential growth. The coefficients are bounded from above by the number of embedded outerplanar graphs with a root edge, which also have exponential growth (this follows from classical enumerative results on planar maps; see [35]). Hence $\rho$ is in $(0,1)$.

To apply Theorem 2.2 for rooted connected outerplanar graphs, we consider the function

$$
H(x, y):=x \exp \left(\frac{Z\left(\mathcal{V} ; y, \hat{C}\left(x^{2}\right)\right)}{y}+\sum_{k \geq 2} \frac{Z\left(\mathcal{V} ; \hat{C}\left(x^{k}\right), \hat{C}\left(x^{2 k}\right)\right)}{k \hat{C}\left(x^{k}\right)}\right)-y .
$$

Observe that Equation (3.5) implies that $H(x, \hat{C}(x))=0$. The difficulty in the application of the singular implicit functions theorem (Thm. 2.2) is the verification of the requirements of this theorem. Hence, to apply Theorem 2.2, we have to check that the dominant singularity of the generating functions for the connected components is determined by its implicit definition (like (3.5)) and not by a singularity of $H(x, y)$. This analysis is the main purpose of the next lemma. Observe that it can be easily applied to other classes of connected unlabeled graphs with known blocks.

Lemma 4.2. The generating function $\hat{C}(x)$ has a singular expansion

$$
\hat{C}(x)=\sum_{k \geq 0} \hat{C}_{k} X^{k}, \quad \text { with } \quad X:=\sqrt{1-\frac{x}{\rho}}, \quad \hat{C}_{0}=\tau, \quad \hat{C}_{1}=-\sqrt{\frac{2 \rho \frac{\partial}{\partial x} H(\rho, \tau)}{\frac{\partial^{2}}{\partial y^{2}} H(\rho, \tau)}}
$$

and constants $\hat{C}_{k}, k \geq 2$, which can be computed from the derivatives of $H(x, y)$ at $(\rho, \tau)$, where $\rho$ is the dominant singularity of $\hat{C}(x)$ and $\tau:=\lim _{x \rightarrow \rho^{-}} \hat{C}(x)$.

Proof. We show that $\hat{C}(x)$ satisfies the conditions of Theorem 2.2 with the function $H(x, y)$ from Equation $(4.1), R:=\min \left(\sqrt{\rho}, \sqrt{\hat{C}^{-1}(\delta)}\right)$, and $S:=\delta$. As a consequence, we obtain the singular expansion (4.2) of $\hat{C}(x)$. 
The conditions $H(0,0)=0$ and $\frac{\partial}{\partial y} H(0,0)=-1$ can be verified easily (note from (3.2) that $Z\left(\mathcal{V} ; y, \hat{C}\left(x^{2}\right)\right) / y$ in $(4.1)$ is well defined for $\left.y=0\right)$. The positivity conditions on the coefficients of $H(x, y)$ follow from the positivity of the coefficients of $Z(\mathcal{V})$. The analyticity domain of $H(x, y)$ is determined by the dominant singularity $\delta$ of $Z(\mathcal{V})$; that is, $H(x, y)$ is analytic for $x$ and $y$ such that $|y|<\delta,\left|x^{l}\right|<\rho$, and $\left|\hat{C}\left(x^{l}\right)\right|<\delta$ for each $l \geq 2$. Since $\hat{C}(x)$ is strictly increasing for positive $x$ and since $\rho<1,\left|\hat{C}\left(x^{l}\right)\right| \leq\left|\hat{C}\left(x^{2}\right)\right|$ for all $l \geq 2$ and $|x|<\rho$. Therefore, $H(x, y)$ is analytic for $|x|<R$ and $|y|<\delta$.

We show next that $\rho<R$ and $\tau<\delta$.

1. We show $\tau \leq \delta$. Let $\tilde{H}(x, y):=H(x, y)+y$. $\tilde{H}(x, y)$ satisfies $\tilde{H}(x, \hat{C}(x))=\hat{C}(x)$ and has the same domain of analyticity as $H(x, y)$. Assume $\tau>\delta$. Then there exists $x_{0}<\rho$ such that $\hat{C}\left(x_{0}\right)=\delta$. Observe that, if $|x|<x_{0}$ then $\left|\hat{C}\left(x^{2}\right)\right| \leq$ $|\hat{C}(x)|<\hat{C}\left(x_{0}\right)=\delta$. Thus $(x, \hat{C}(x))$ is in the analyticity domain of $\tilde{H}(x, y)$, so that $\tilde{H}(x, \hat{C}(x))=\hat{C}(x)$. By continuity we obtain $\tilde{H}\left(x_{0}, \hat{C}\left(x_{0}\right)\right)=\hat{C}\left(x_{0}\right)$. We have now the contradiction that $\hat{C}(x)$ is analytic at $x_{0}$ since $x_{0}<\rho$, whereas $\tilde{H}(x, \hat{C}(x))$ is singular at $x_{0}$ because $\hat{C}\left(x_{0}\right)=\delta$.

2. From 1 we know that $\tau \leq \delta$, i.e., $\rho \leq \hat{C}^{-1}(\delta)$. Hence $R=\sqrt{\rho}>\rho$.

3. It remains to prove that $\tau<\delta$. Assume $\tau=\delta$. Observe from (3.2) and (3.5) that

$$
\hat{C}(x)=x \exp (\Psi(\hat{C}(x))+A(x))
$$

where $\Psi(y)=1 / 8\left(1+y-\sqrt{1-6 y+y^{2}}\right)$ has a dominant singularity at $y=\delta$ and

$$
A(x)=\frac{\hat{C}(x)+\hat{C}\left(x^{2}\right)}{8 \hat{C}\left(x^{2}\right)^{2}}\left(1-3 \hat{C}\left(x^{2}\right)-\sqrt{\hat{C}\left(x^{2}\right)^{2}-6 \hat{C}\left(x^{2}\right)+1}\right)+\sum_{k \geq 2} \frac{Z\left(\mathcal{V} ; \hat{C}\left(x^{k}\right), \hat{C}\left(x^{2 k}\right)\right)}{k \hat{C}\left(x^{k}\right)}
$$

is analytic for $|x|<\rho$ and having nonnegative coefficients (because the double of the first summand of $A(x)$ is the generating function for reflective vertex rooted dissections [37]). Hence, for $0<x<\rho, \hat{C}^{\prime}(x) \geq \hat{C}^{\prime}(x) \Psi^{\prime}(\hat{C}(x)) \hat{C}(x)$, so that $\Psi^{\prime}(\hat{C}(x)) \leq 1 / \hat{C}(x)$. Thus, $\Psi^{\prime}(\hat{C}(x))$ is bounded when $x \rightarrow \rho^{-}$, which contradicts the fact that $\lim _{y \rightarrow \delta^{-}} \Psi^{\prime}(y)=+\infty$.

Thus, $H(x, y)$ is analytic at $(\rho, \tau)$ and $H(\rho, \tau)=0$ is satisfied. As pointed out before, the dominant singularity $\rho$ of $\hat{C}(x)$ is determined either by a singularity in a component of Equation (3.5), or by a non-uniqueness in the definition of $\hat{C}(x)$ by Equation (3.5). The relation $\tau<\delta$ excludes the first case, so that the singularity is caused by a nonuniqueness of the inversion. Hence, the derivative of $H(x, y)$ with respect to $y$ has to vanish at $(x, y)=(\rho, \tau)$, since otherwise the implicit function theorem ensures a (unique) analytic continuation of $\hat{C}(x)$ at $x=\rho$. Therefore, the first two equations of (2.5) are 
satisfied. Furthermore, it is easily verified that

$$
\begin{aligned}
\left.\frac{\partial^{2}}{\partial y^{2}} H(x, y)\right|_{(x, y)=(\rho, \tau)} & =\frac{1}{\tau}+\left.\frac{\partial^{2}}{\partial s_{1}^{2}} \frac{Z\left(\mathcal{V} ; s_{1}, \hat{C}\left(\rho^{2}\right)\right)}{s_{1}}\right|_{s_{1}=\tau}=\frac{1}{\tau}+\frac{\tau}{\left(\tau^{2}-6 \tau+1\right)^{3 / 2}}, \\
\left.\frac{\partial}{\partial x} H(x, y)\right|_{(x, y)=(\rho, \tau)} & =\tau\left(\frac{1}{\rho}+\frac{\partial}{\partial x} \frac{Z\left(\mathcal{V} ; \tau, \hat{C}\left(x^{2}\right)\right)}{\tau}+\left.\sum_{k \geq 2} \frac{Z\left(\mathcal{V} ; \hat{C}\left(x^{k}\right), \hat{C}\left(x^{2 k}\right)\right)}{k \hat{C}\left(x^{k}\right)}\right|_{x=\rho}\right) .
\end{aligned}
$$

From $0<\tau<\delta$ and the fact that the derivative in $\frac{\partial}{\partial x} H(\rho, \tau)$ is a derivative of a formal power series with positive coefficients evaluated at $\rho>0$, it follows that both derivatives are strictly positive and hence do not vanish.

Finally, the aperiodicity of $\hat{C}(x)$ is easily seen from the fact that $\hat{c}_{1} \neq 0$ and $\hat{c}_{2} \neq 0$.

Theorem 4.3 (asymptotic number of connected outerplanar graphs). The function $C(x)$ has a singular expansion of the form

$$
C(x)=C(\rho)+\sum_{k \geq 2} C_{k} X^{k}, \quad X:=\sqrt{1-\frac{x}{\rho}},
$$

with constants $C_{k}, k \geq 2$, which can be computed from the constants $\hat{C}_{k}$, and with $\rho$ as in Lemma 4.2. Hence, $c_{n} \sim \frac{3}{4 \sqrt{\pi}} C_{3} n^{-5 / 2} \rho^{-n}$.

Proof. Recall Formula (3.6) for the ordinary generating function for connected outerplanar graphs. Since $\tau<\delta$, it is clear that the dominant singularity of $C(x)$ is the same as $\hat{C}(x)$ [14, Cha. VI.9]. The singular expansion of $C(x)$ around $\rho$ can then be obtained by injecting the singular expansion of $\hat{C}(x)$ into Formula (3.6):

$$
\begin{aligned}
C(x)= & \sum_{k \geq 0} \hat{C}_{k} X^{k}+Z\left(\mathcal{D} ; \sum_{k \geq 0} \hat{C}_{k} X^{k}, \hat{C}\left(\rho^{2}\left(1-X^{2}\right)^{2}\right), \hat{C}\left(\rho^{3}\left(1-X^{2}\right)^{3}\right), \ldots\right) \\
& -Z\left(\mathcal{V} ; \sum_{k \geq 0} \hat{C}_{k} X^{k}, \hat{C}\left(\rho^{2}\left(1-X^{2}\right)^{2}\right), \hat{C}\left(\rho^{3}\left(1-X^{2}\right)^{3}\right), \ldots\right) .
\end{aligned}
$$

Developing it in terms of $X$ (at $X=0$ ) gives a singular expansion $C(x)=\sum_{k \geq 0} C_{k} X^{k}$.

It remains to check that $C_{1}=0$ and $C_{3} \neq 0$. From (4.4) it is clear that

$$
C_{1}=\hat{C}_{1}+\left.\hat{C}_{1} \frac{\partial}{\partial s_{1}} Z(\mathcal{D})\right|_{\left(s_{1}, s_{2}\right)=\left(\tau, \hat{C}\left(\rho^{2}\right)\right)}-\left.\hat{C}_{1} \frac{\partial}{\partial s_{1}} Z(\mathcal{V})\right|_{\left(s_{1}, s_{2}\right)=\left(\tau, \hat{C}\left(\rho^{2}\right)\right)} .
$$

From $(2.2)$ we know $s_{1} \frac{\partial}{\partial s_{1}} Z(\mathcal{D})=Z(\mathcal{V})$, so that

$$
C_{1}=\left.\hat{C}_{1}\left(1+\frac{Z(\mathcal{V})}{s_{1}}-\frac{\partial}{\partial s_{1}} Z(\mathcal{V})\right)\right|_{\left(s_{1}, s_{2}\right)=\left(\tau, \hat{C}\left(\rho^{2}\right)\right)}
$$


On the other hand, Equation (4.1) implies that

$$
\frac{\partial}{\partial y} H(x, y)=(H(x, y)+y)\left(\frac{1}{y} \frac{\partial}{\partial s_{1}} Z\left(\mathcal{V} ; y, \hat{C}\left(x^{2}\right)\right)-\frac{1}{y^{2}} Z\left(\mathcal{V} ; y, \hat{C}\left(x^{2}\right)\right)\right)-1 .
$$

By (2.5) and Lemma 4.2, $0=\frac{\partial}{\partial y} H(\rho, \tau)=\frac{\partial}{\partial s_{1}} Z(\mathcal{V})-\frac{1}{s_{1}} Z(\mathcal{V})-\left.1\right|_{\left(s_{1}, s_{2}\right)=\left(\tau, \hat{C}\left(\rho^{2}\right)\right)}$. Thus, $C_{1}=0$. Assume $C_{3}=0$. Then the expansion (4.3) yields $c_{n} \sim O\left(n^{-k / 2-1}\right) \rho^{-n}$ for some odd number $k \geq 5$. This contradicts $n c_{n} \geq \hat{c}_{n} \sim-\hat{C}_{1} /(2 \sqrt{\pi}) n^{-3 / 2} \rho^{-n}$ (by Lemma 4.2).

Theorem 4.4 (asymptotic number of outerplanar graphs). The function $G(x)$ has a singular expansion of the form

$$
G(x)=G(\rho)+\sum_{k \geq 2} G_{k} X^{k}, \quad X:=\sqrt{1-\frac{x}{\rho}},
$$

where $\rho$ is as in Lemma 4.2, and where the constants $G_{k}, k \geq 2$, can be computed from the constants $C_{k}$, in particular $G_{3}=G(\rho) C_{3}$. Hence, $g_{n} \sim \frac{3}{4 \sqrt{\pi}} G_{3} n^{-5 / 2} \rho^{-n}$.

Proof. Recall Formula (3.7) for the ordinary generating function for outerplanar graphs. As the exponential function $\exp (\cdot)$ is regular, the dominant singularity of $G(x)$ is the same as $C(x)$. Replacing $C(x)$ by its singular expansion (4.3) and $x^{k}$ by $\rho^{k}\left(1-X^{2}\right)^{k}$ for $k \geq 2$, we get

$$
G(x)=\exp \left(C(\rho)+\sum_{k \geq 2} C_{k} X^{k}+\sum_{k \geq 2} \frac{1}{k} C\left(\rho^{k}\left(1-X^{2}\right)^{k}\right)\right),
$$

from which the singular expansion of $G(x)$ can be computed. Then, by Lemma 2.1 we derive the asymptotic estimate of $g_{n}$.

Finally, using the same techniques as for the general case, we can compute the asymptotic estimate of bipartite outerplanar graphs, given in Theorem 1.5.

\subsection{Numerical approximation of the growth constants}

The computation of analytic expressions for growth constants has not been possible for some classes of unlabeled structures that are even simpler than outerplanar graphs, for example, for unembedded trees, see [14, Cha. VII.5] and [28]. Nevertheless, we can simplify the problem by reducing it to one variable, and provide numerical estimates of the growth constants. With Formula (4.5) for $\frac{\partial}{\partial y} H(\rho, \tau)$ and the explicit formula for $Z(\mathcal{V})$ from Corollary 3.2 the equation $\frac{\partial}{\partial y} H(\rho, \tau)=0$ becomes

$$
\tau\left(1+\hat{C}\left(\rho^{2}\right)\left(\hat{C}\left(\rho^{2}\right)-3\right)-\frac{\hat{C}\left(\rho^{2}\right)^{2}(\tau-3)}{\sqrt{\tau^{2}-6 \tau+1}}-\sqrt{\hat{C}\left(\rho^{2}\right)^{2}-6 \hat{C}\left(\rho^{2}\right)+1}\right)=8 \hat{C}\left(\rho^{2}\right)^{2}
$$


By introducing two new variables for the square-root terms in Equation (4.6), it can be reformulated as a system of three polynomial equations, regarding $\hat{C}\left(\rho^{2}\right)$ as a fixed value. With algebraic elimination [14, App. B.1], this system can be reduced to one polynomial equation of degree 8 in $\tau$ with coefficients $p_{i}(\rho)$ (depending on $\hat{C}\left(\rho^{2}\right)$ ), $i=0, \ldots, 8$,

$p_{0}(\rho)+p_{1}(\rho) \tau+p_{2}(\rho) \tau^{2}+p_{3}(\rho) \tau^{3}+p_{4}(\rho) \tau^{4}+p_{5}(\rho) \tau^{5}+p_{6}(\rho) \tau^{6}+p_{7}(\rho) \tau^{7}+p_{8}(\rho) \tau^{8}=0$

(see [37] for details). The solutions of (4.7) do not need to satisfy Equation (4.6), but every $\tau$ that is a solution of (4.6) is also a solution of (4.7). We denote the solutions of (4.7) by $\tau_{1}(\rho), \ldots, \tau_{8}(\rho)$. It remains to solve the equations $H\left(\rho, \tau_{i}(\rho)\right)=0, i=1, \ldots, 8$, and to pick the correct solution $\rho$. Since $H(x, y)$ depends on $\hat{C}(x)$, which we do not know explicitly, and since it includes also an infinite sum that we were not able to simplify, we can only approximate the solutions of $H\left(\rho, \tau_{i}(\rho)\right)=0$ by truncating the infinite sum in $H(x, y)$ at some index $m$ and replacing $\hat{C}(x)$ with $\hat{C}^{[m]}(x):=\sum_{n=1}^{m} \hat{c}_{n} x^{n}$ for known coefficients $\hat{c}_{1}, \ldots, \hat{c}_{m}$. That is, we search for roots of the functions

$$
\tilde{H}_{i}^{[m]}(\rho):=\rho \exp \left(\frac{Z\left(\mathcal{V} ; \tau_{i}(\rho), \hat{C}^{[m]}\left(\rho^{2}\right)\right)}{\tau_{i}(\rho)}+\sum_{k=2}^{m} \frac{Z\left(\mathcal{V} ; \hat{C}^{[m]}\left(\rho^{k}\right), \hat{C}^{[m]}\left(\rho^{2 k}\right)\right)}{k \hat{C}^{[m]}\left(\rho^{k}\right)}\right)-\tau_{i}(\rho),
$$

$i=1, \ldots, 8$, in the interval $(0,1)$. We solved the equation $\tilde{H}_{i}^{[m]}(\rho)=0$ for $m=25$ numerically and obtained 8 solutions. From these 8 solutions we selected the real number of smallest absolute value (in order to obtain a dominant singularity, see also Pringsheim's theorem [14, Thm. IV.6]). This gives the estimates

$$
\rho \approx 0.1332694 \text { and } \tau \approx 0.1707560 .
$$

The residuals in the equations $\tilde{H}_{i}^{[m]}(\rho, \tau)=0$ and $\frac{\partial}{\partial y} \tilde{H}_{i}^{[m]}(\rho, \tau)=0$ have an order of $10^{-58}$. Table 1 shows approximations of $\rho$ for several values of $m$.

\begin{tabular}{|c|c|}
\hline$m$ & \multicolumn{1}{|c|}{ approximation of $\rho$} \\
\hline \hline 1 & $\mathbf{0 . 1 3 4 6 1 8 7 6 8 8 6 1 1 0 1 8 1 3 6 9 \ldots}$ \\
\hline 4 & $\mathbf{0 . 1 3 3 2} 7064317786556821 \ldots$ \\
\hline 8 & $\mathbf{0 . 1 3 3 2 6 9 4 3 2} 88029243729 \ldots$ \\
\hline 16 & $\mathbf{0 . 1 3 3 2 6 9 4 3 2 6 6 7 4 4 6 8 2 0 7 1 \ldots}$ \\
\hline 25 & $\mathbf{0 . 1 3 3 2 6 9 4 3 2 6 6 7 4 4 6 8 0 9 4 4 \ldots}$ \\
\hline
\end{tabular}

Table 1: The accuracy is improved by increasing the order of truncation.

We can now estimate the coefficients in the singular expansions of $\hat{C}(x), C(x)$, and $G(x)$. In particular $\hat{C}_{1} \approx-0.0255905, C_{3} \approx 0.0179720$, and $G_{3} \approx 0.0215044$.

The growth constant for bipartite outerplanar graphs can also be estimated in the same way as $\rho$, and we get $\rho_{b} \approx 0.218475$ (see [37] for details). 


\section{A random outerplanar graph}

This section investigates typical properties of a random outerplanar graph, i.e., a graph chosen uniformly at random among all unlabeled outerplanar graphs with $n$ vertices, as $n$ tends to infinity. We first discuss the probability of connectedness, the number and type of components, and the number of isolated vertices, in a random outerplanar graph. Next, we investigate the distribution of the number of edges in a random outerplanar graph. Finally, we discuss the chromatic number of a random outerplanar graph.

\subsection{Connectedness, components, and isolated vertices}

We start with the proof of Theorem 1.3 (1).

Proof of Theorem 1.3 (1). The probability that a random outerplanar graph on $n$ vertices is connected is exactly $c_{n} / g_{n}$. The asymptotic estimates for $c_{n}$ and $g_{n}$ from Theorem 4.3 and Theorem 4.4 yield $c_{n} / g_{n} \sim C_{3} / G_{3} \approx 0.845721$.

The number of components can be studied by augmenting the generating function for outerplanar graphs with a variable that counts the number of components.

Proof of Theorem 1.3 (2). Let $\kappa_{n}$ denote the number of components in a random outerplanar graph on $n$ vertices and let $G(x, u):=\exp \left(\sum_{k \geq 1} \frac{1}{k} u^{k} C\left(x^{k}\right)\right)$ be the generating function for outerplanar graphs, where the additional variable $u$ marks the number of components. Thus, the probability that an outerplanar graph has $k$ components is $\mathbb{P}\left[\kappa_{n}=k\right]=\left[x^{n} u^{k}\right] G(x, u) / g_{n}$, and the expected number of components is

$$
\mathbb{E}\left[\kappa_{n}\right]=\frac{1}{g_{n}} \sum_{k \geq 1} k\left[x^{n} u^{k}\right] G(x, u)=\frac{1}{g_{n}}\left[x^{n}\right] \frac{\partial}{\partial u} G(x, 1)=\frac{1}{g_{n}}\left[x^{n}\right] G(x) \sum_{k \geq 1} C\left(x^{k}\right) .
$$

Now split $G(x) \sum_{k \geq 1} C\left(x^{k}\right)$ as $G(x) C(x)+G(x) \sum_{k \geq 2} C\left(x^{k}\right)$. As the singularity $\rho$ of $C(x)$ (and of $G(x)$ ) is smaller than 1 and $C(x)=O(x)$ as $x \rightarrow 0$, the series $A(x):=\sum_{k \geq 2} C\left(x^{k}\right)$ is regular at $\rho$. Consequently,

$$
\left[x^{n}\right] G(x) A(x) \sim\left[x^{n}\right] G(x) A(\rho) \sim G_{3} \frac{A(\rho)}{\Gamma(-3 / 2)} n^{-5 / 2} \rho^{-n} .
$$

Next, the singular development of $G(x) C(x)$ starts as

$$
G(x) C(x)=G(\rho) C(\rho)+\left(C_{2} G(\rho)+G_{2} C(\rho)\right) X^{2}+\left(C_{3} G(\rho)+G_{3} C(\rho)\right) X^{3}+O\left(X^{4}\right) .
$$

Hence, the leading term is in $X^{3}$. Since $G_{3}=G(\rho) C_{3}$, we obtain $\left[X^{3}\right] G(x) C(x)=$ $G_{3}(1+C(\rho))$, so that

$$
\left[x^{n}\right] G(x) C(x) \sim G_{3}(1+C(\rho)) \frac{1}{\Gamma(-3 / 2)} n^{-5 / 2} \rho^{-n} .
$$


Summing (5.1) and (5.2) and dividing by $g_{n} \sim G_{3} \Gamma(-3 / 2)^{-1} n^{-5 / 2} \rho^{-n}$, we obtain

$$
\mathbb{E}\left[\kappa_{n}\right] \sim 1+\sum_{k \geq 1} C\left(\rho^{k}\right) \approx 1.17847
$$

where the value 1.17847 was computed by replacing infinite sums in $\mathbb{E}\left[\kappa_{n}\right]$ and the involved generating functions by finite sums and using the approximated value for $\rho$.

Given a family $\mathcal{A}$ of connected outerplanar graphs, we can make the following statements about the probability that a random outerplanar graph has exactly $k$ components in $\mathcal{A}$. Denote the number of graphs in $\mathcal{A}$ that have exactly $n$ vertices by $a_{n}$, and let $A(x):=\sum_{n} a_{n} x^{n}$.

Theorem 5.1. Given an outerplanar graph $G$ with $n$ vertices, let $\kappa_{n}^{\mathcal{A}}$ be the number of connected components of $G$ belonging to $\mathcal{A}$. If the radius of convergence $\alpha$ of $A(x)$ is strictly larger than $\rho$, that is, $a_{n}$ is exponentially smaller than $c_{n}$, then the probability that a random outerplanar graph with $n$ vertices has exactly $k \geq 0$ components belonging to $\mathcal{A}$ converges to a discrete law:

$$
\mathbb{P}\left[\kappa_{n}^{\mathcal{A}}=k\right] \sim Z\left(S_{k} ; A(\rho)\right) \exp \left(-\sum_{r \geq 1} \frac{1}{r} A\left(\rho^{r}\right)\right),
$$

where $S_{k}$ is the symmetric group on $k$ vertices [21]. The expected number of components belonging to $\mathcal{A}$ in a random outerplanar graph with $n$ vertices is $\mathbb{E}\left[\kappa_{n}^{\mathcal{A}}\right] \sim \sum_{r \geq 1} A\left(\rho^{r}\right)$.

Proof. Let $G^{\mathcal{A}}(x, u)$ be the generating function for outerplanar graphs, where the additional variable $u$ marks the number of components belonging to $\mathcal{A}$,

$$
\begin{aligned}
G^{\mathcal{A}}(x, u) & :=\exp \left(\sum_{k \geq 1} \frac{1}{k}\left(u^{k} A\left(x^{k}\right)+\left(C\left(x^{k}\right)-A\left(x^{k}\right)\right)\right)\right) \\
& =G(x) \exp \left(\sum_{k \geq 1} \frac{u^{k}-1}{k} A\left(x^{k}\right)\right) .
\end{aligned}
$$

Hence, $\mathbb{P}\left[\kappa_{n}^{\mathcal{A}}=k\right]=\left[x^{n} u^{k}\right] G^{\mathcal{A}}(x, u) / g_{n}$. Since $A(x)$ is analytic at $\rho$, the dominant singularity of $G^{\mathcal{A}}(x, u)$ for fixed $u$ is determined by $G(x)$. Thus,

$$
\left[x^{n} u^{k}\right] G^{\mathcal{A}}(x, u) \underset{n \rightarrow \infty}{\sim}\left[u^{k}\right] \exp \left(\sum_{k \geq 1} \frac{u^{k}-1}{k} A\left(\rho^{k}\right)\right)\left[x^{n}\right] G(x),
$$

i.e.,

$$
\mathbb{P}\left[\kappa_{n}^{\mathcal{A}}=k\right] \underset{n \rightarrow \infty}{\sim}\left[u^{k}\right] \exp \left(\sum_{k \geq 1} \frac{u^{k}-1}{k} A\left(\rho^{k}\right)\right)=Z\left(S_{k} ; A(\rho)\right) \exp \left(-\sum_{k \geq 1} \frac{1}{k} A\left(\rho^{k}\right)\right) .
$$


For the expectation, we again use

$$
\mathbb{E}\left[\kappa_{n}^{\mathcal{A}}\right]=\frac{1}{g_{n}}\left[x^{n}\right] \frac{\partial}{\partial u} G^{\mathcal{A}}(x, 1)=\frac{1}{g_{n}}\left[x^{n}\right] G(x) \sum_{k \geq 1} A\left(x^{k}\right) .
$$

The statement follows from the analyticity of $A(x)$ at $\rho$ and Theorem 4.4.

The asymptotic distribution of the number of isolated vertices in a random outerplanar graph can now be easily computed, as stated in Theorem 1.3 (3).

Proof of Theorem 1.3 (3). Let $\mathcal{A}$ be the family consisting of the graph that is a single vertex, i.e., $A(x)=x$. By Theorem 5.1, $\mathbb{P}\left[\kappa_{n}^{\mathcal{A}}=k\right] \sim \rho^{k}(1-\rho)$, since $Z\left(S_{k} ; A(\rho)\right)=\rho^{k}$ and $-\sum_{r} \frac{1}{r} A\left(\rho^{r}\right)=\log (1-\rho)$. Hence, the distribution of the number of isolated vertices $\kappa_{n}^{\mathcal{A}}$ follows asymptotically a geometric law with parameter $\rho$.

Other consequences of Theorem 5.1 concern the number of two-connected components and the number of bipartite components in a random outerplanar graph.

Corollary 5.2 (two-connected components). In a random outerplanar graph, the expected number of connected components that are two-connected is asymptotically given by $\sum_{k \geq 1} D\left(\rho^{k}\right) \approx 0.175054$.

Proof. Let $\mathcal{A}:=\mathcal{D}$ be the family of dissections, $A(x)=D(x)$. The radius of convergence of $D(x)$ is $\delta>\rho$ (Lemma 4.2). Hence, by Theorem 5.1, $\mathbb{E}\left[\kappa_{n}^{\mathcal{D}}\right]=\sum_{k \geq 1} D\left(\rho^{k}\right)$.

Corollary 5.3 (number of bipartite components). In a random outerplanar graph, the expected number of connected components that are bipartite is asymptotically given by $\sum_{k \geq 1} C_{b}\left(\rho^{k}\right) \approx 0.175427$, where $C_{b}(x)$ is the generating function for bipartite connected outerplanar graphs.

Proof. We apply Theorem 5.1 with $\mathcal{A}=\mathcal{C}_{b}$.

\section{$5.2 \quad$ Number of edges}

In this section, we analyze the distribution of the number of edges in a random outerplanar graph. To do this, we add a variable $y$ whose power (in the cycle index sums and generating functions) indicates the number of edges. For a graph $G$ on $n$ vertices and $m$ edges, and with the automorphism group $\Gamma(G)$ (acting on the vertices), we define

$$
Z\left(G ; s_{1}, s_{2}, \ldots ; y\right):=Z\left(\Gamma(G) ; s_{1}, s_{2}, \ldots ; y\right):=y^{m} \frac{1}{|\Gamma(G)|} \sum_{\alpha \in \Gamma(G)} \prod_{k=1}^{n} s_{k}^{j_{k}(\alpha)}
$$

Taking the number of edges into account in the calculations of Section 3, the cycle index sums for all encountered families of outerplanar graphs can be derived with the additional variable $y$, and further the coefficients counting outerplanar graphs with respect to the 
number of vertices and the number of edges can be computed in polynomial time, see [37] for details.

With the help of Theorem 2.3, we can prove Theorem 1.4 giving the limit distributions of the number of edges in a random dissection and in a random outerplanar graph, respectively.

Proof of Theorem 1.4. The limit distribution of the number of edges in a two-connected unlabeled outerplanar graph coincides with the labeled case since the equation that determines the dominant singularity is the same, so we refer to [4].

Next we determine the distribution of the number of edges in a rooted connected outerplanar graph. The generating function $\hat{C}(x, y)$ is implicitly defined by

$$
\hat{C}(x, y)=x \exp \left(\sum_{k \geq 1} \frac{Z\left(\mathcal{V} ; \hat{C}\left(x^{k}, y^{k}\right), \hat{C}\left(x^{2 k}, y^{2 k}\right) ; y^{k}\right)}{k \hat{C}\left(x^{k}, y^{k}\right)}\right) .
$$

In order to apply the singular implicit functions theorem 2.2 to the function $x \mapsto \hat{C}(x, y)$ with a fixed $y$ close to 1 , we define

$H_{y}(x, z):=x \exp \left(\frac{Z\left(\mathcal{V} ; z, \hat{C}\left(x^{2}, y^{2}\right) ; y\right)}{z}+\sum_{k \geq 2} \frac{Z\left(\mathcal{V} ; \hat{C}\left(x^{k}, y^{k}\right), \hat{C}\left(x^{2 k}, y^{2 k}\right) ; y^{k}\right)}{k \hat{C}\left(x^{k}, y^{k}\right)}\right)-z$

and search for a solution $(x, z)=(\rho(y), \tau(y))$ of the system

$$
H_{y}(x, z)=0, \quad \frac{\partial}{\partial z} H_{y}(x, z)=0,
$$

such that $(\rho(y), \tau(y))$ is in the analyticity domain of $H_{y}(x, z)$.

For $y=1,(5.3)$ is satisfied by $x=\rho, z=\tau$ (Lemma 4.2). The classical implicit functions theorem, applied to the system (5.3), ensures that this solution can be extended to a curve $(\rho(y), \tau(y))$ for $y$ close to 1 , where the functions $\rho(y)$ and $\tau(y)$ are analytic in a neighbourhood of 1 . Therefor, it remains to check that the determinant of the Jacobian of system (5.3), with respect to $x$ and $z$,

$$
\left(\begin{array}{cc}
\frac{\partial}{\partial x} H_{y}(x, z) & \frac{\partial}{\partial z} H_{y}(x, z) \\
\frac{\partial}{\partial x} \frac{\partial}{\partial z} H_{y}(x, z) & \frac{\partial}{\partial z} \frac{\partial}{\partial z} H_{y}(x, z)
\end{array}\right)
$$

does not vanish at $y=1$ and $(x, z)=(\rho(1), \tau(1))$. This is clear, since from Lemma 4.2 we have $\frac{\partial}{\partial z} H_{1}(\rho(1), \tau(1))=0, \frac{\partial}{\partial x} H_{1}(\rho(1), \tau(1)) \neq 0$, and $\frac{\partial^{2}}{\partial z^{2}} H_{1}(\rho(1), \tau(1)) \neq 0$. Hence, for $y$ close to 1 , there exist analytic functions $\rho(y)$ and $\tau(y)$ such that

$$
\begin{aligned}
H_{y}(\rho(y), \tau(y)) & =0, \quad \frac{\partial}{\partial z} H_{y}(\rho(y), \tau(y))=0, \\
\frac{\partial^{2}}{\partial z^{2}} H_{y}(\rho(y), \tau(y)) & \neq 0, \quad \frac{\partial}{\partial x} H_{y}(\rho(y), \tau(y)) \neq 0 .
\end{aligned}
$$


In addition, by analyticity of $H_{1}(x, z)$ at $(\rho, \tau)$, the solution $(\rho(y), \tau(y))$ is in the analyticity domain of $H_{y}(x, z)$ (for $y$ close to 1 ). Next, the singular implicit functions theorem 2.2 yields a singular expansion $\hat{C}(x, y)=\sum_{k \geq 0} \hat{C}_{k}(y)(\sqrt{1-x / \rho(y)})^{k}$ with coefficients $\hat{C}_{k}(y)$ analytic at $y=1$ and verifying $\hat{C}_{1}(y) \neq 0$ for $y$ close to 1 .

To find $\rho^{\prime}(1)$ and $\rho^{\prime \prime}(1)$ we compute the first and second derivatives of the first two equations in (5.4) with respect to $y$, and express $\rho^{\prime}(y)$ and $\rho^{\prime \prime}(y)$ in terms of $\rho(y), \tau(y)$, and the partial derivatives of $H_{y}(x, z)$ at $(x, z)=(\rho(y), \tau(y))$. Using the approximated values from Section 4.2 we obtain $\rho^{\prime}(1) \approx-0.206426, \rho^{\prime \prime}(1) \approx 0.495849$, and $-\frac{\rho^{\prime \prime}(1)}{\rho(1)}-\frac{\rho^{\prime}(1)}{\rho(1)}+$ $\left(\frac{\rho^{\prime}(1)}{\rho(1)}\right)^{2} \approx 0.227504 \neq 0$. Theorem 2.3 implies that the distribution of the number of edges in a random rooted connected outerplanar graph with $n$ vertices asymptotically follows a Gaussian law with mean $\mu n$ and variance $\sigma^{2} n$, where $\mu=-\frac{\rho^{\prime}(1)}{\rho(1)} \approx 1.54894$ and $\sigma^{2} \approx$ 0.227504 . The same holds for unrooted connected outerplanar graphs and for outerplanar graphs, since their generating functions have the same dominant singularity.

\subsection{Chromatic number}

In this section, we investigate the colouring of a random outerplanar graph. We first observe that every outerplanar graph is 3-colourable. Next, we compute the asymptotic fraction of 2-colourable outerplanar graphs among all outerplanar graphs. Theorems 1.2 and 1.5 imply that the fraction of bipartite outerplanar graphs on $n$ vertices among all outerplanar graphs on $n$ vertices is

$$
\frac{\left(g_{b}\right)_{n}}{g_{n}} \sim c_{\chi} \rho_{\chi}^{n},
$$

where $c_{\chi}=\frac{g}{b}>0$ and $\rho_{\chi}=\frac{\rho_{b}^{-1}}{\rho^{-1}} \approx 0.60999<1$. That is, the probability that the chromatic number of a random outerplanar graph is two tends to 0 exponentially fast. This completes the proof of Theorem 1.6.

\section{Concluding remarks}

A summary of the estimated growth constants and other parameters for unlabeled outerplanar graphs is presented in Table 2. For comparison we also include the corresponding labeled quantities derived in [4]. As mentioned before, in the two-connected case the estimated quantities for the unlabeled and labeled structures do not differ, since the number of dissections with a non-trivial symmetry is exponentially small.

Our results show that the combination of enumeration by cycle index sums and singularity analysis is a powerful tool for the analysis of unlabeled structures. Hence, an interesting direction for further research is the extension of the applied techniques to other classes of unlabeled structures, e.g., series-parallel graphs or planar graphs. Thereby, a first challenge is the construction of cycle index sums for the two-connected components. While we used the unique embedding of two-connected components of outerplanar 


\begin{tabular}{l||c|c|c}
\multicolumn{1}{c||}{} & dissections & \multicolumn{2}{c}{ outerplanar graphs } \\
& unlabeled or labeled & unlabeled & labeled \\
\hline \hline growth constant & $\delta^{-1} \approx 5.82843$ & $\rho^{-1} \approx 7.50360$ & $\lambda^{-1} \approx 7.32098$ \\
\hline $\mathbb{P}$ [connectivity] & 1 & 0.845721 & 0.861667 \\
$\mathbb{E}$ [nr. of components] & 1 & 1.17847 & - \\
\hline distr. nr. of isolated vertices & Dirac & Geom $(\rho)$ & Poiss $(\lambda)$ \\
$\mathbb{E}$ [nr. of isolated vertices] & 0 & 0.153761 & 0.136593 \\
\hline distr. of nr. of edges & Gaussian & Gaussian & Gaussian \\
$\mathbb{E}$ [nr. of edges] & $1.70711 n$ & $1.54894 n$ & $1.56251 n$ \\
$\mathbb{V}$ [nr. of edges] & $0.176777 n$ & $0.227504 n$ & $0.223992 n$ \\
\hline chromatic number & 3 & 3 & 3
\end{tabular}

Table 2: Summary of growth constants, typical properties, and limit laws for unlabeled and labeled dissections and outerplanar graphs.

graphs in our enumeration, this is no longer possible for series-parallel graphs or planar graphs. Thus, although the case of three-connected unlabeled planar graphs is well studied $[1,15,23,35]$, not much is known about two-connected unlabeled planar graphs. For two-connected series-parallel graphs, cycle index sums are known [38], but their asymptotic expansions haven't been studied yet.

Another direction for further research is the extension of our analysis of properties of random outerplanar graphs to other natural parameters. E.g., while Theorem 5.1 allows to compute the distribution of the number of components of a specific type, an interesting question is the analysis of the number of copies of a fixed outerplanar graph. For this purpose, one way to approach this problem is to derive cycle index sums of dissections or connected components with an extra variable marking the number of copies of the given graph, to extend these formulas to outerplanar graphs by following the degree of connectivity, and to apply singularity analysis in the spirit of Theorem 2.3 and [14, Cha. IX].

\section{References}

[1] C. Banderier, P. Flajolet, G. Schaeffer, and M. Soria, Random maps, coalescing saddles, singularity analysis, and Airy phenomena, Random Structures and Algorithms, 19 (2001), $194-246$.

[2] E. A. Bender, Z. Gao, and N. C. Wormald, The number of labeled 2-connected planar graphs, The Electronic Journal of Combinatorics, 9 (2002), \#43.

[3] F. Bergeron, G. Labelle, P. Leroux, Combinatorial Species and Tree-like Structures, Cambridge University Press, Cambridge (1998)

[4] M. Bodirsky, O. Giménez, M. Kang, and M. Noy, The asymptotic number of outerplanar graphs and series-parallel graphs, in the Proceedings of European Conference on Combinatorics, Graph Theory, and Applications (EuroComb05), DMTCS Proceedings Volume AE (2005), $383-388$. 
[5] M. Bodirsky, C. Gröpl, D. Johannsen, and M. Kang, A direct decomposition of 3-connected planar graphs, in the Proceedings of the 17th Annual International Conference on Formal Power Series and Algebraic Combinatorics (FPSAC'05), 2005.

[6] M. Bodirsky, C. Gröpl, and M. Kang, Decomposing, counting, and generating unlabeled cubic planar graphs, submitted (2004).

[7] M. Bodirsky, C. Gröpl, and M. Kang, Sampling unlabeled biconnected planar graphs, in the Proceedings of the 16th Annual International Symposium on Algorithms and Computation (ISAAC'05), 2005, Springer LNCS 3827, 593 - 603.

[8] M. Bodirsky, C. Gröpl, and M. Kang, Generating labeled planar graphs uniformly at random, in the Proceedings of the 13th International Colloquium on Automata, Languages and Programming (ICALP'03), 2003, Springer LNCS 2719, 1095 - 1107.

[9] M. Bodirsky and M. Kang, Generating outerplanar graphs uniformly at random, accepted for publication in Combinatorics, Probability, and Computation (2003).

[10] G. Chartrand and F. Harary, Planar permutation graphs, Ann. Inst. Henry Poincaré, Nouv. Sér., Sect. B3 (1967), $433-438$.

[11] A. Denise, M. Vasconcellos, and D. Welsh, The random planar graph, Congressus Numerantium, 113 (1996), 61-79.

[12] S. Finch, Two asymptotic series (2003), available online at http://pauillac.inria.fr/ algo/csolve/asym.pdf.

[13] P. Flajolet and M. Noy, Analytic combinatorics of non-crossing configurations, Discrete Mathematics, 204 (1999), 203-229

[14] P. Flajolet and R. Sedgewick, Analytic combinatorics, Web Edition (version of 14 February 2007, last updated on 3 May 2007), available online at http://algo.inria.fr/flajolet/ Publications/book061023.pdf.

[15] É. Fusy, Counting unrooted maps using tree-decomposition, in the Proceedings of the 17th International Conference on Formal Power Series and Algebraic Combinatorics (FPSAC05).

[16] É. Fusy, Quadratic exact-size and linear approximate-size random sampling of planar graphs, in the Proceedings of the International Conference on the Analysis of Algorithms (AofA'05), DMTCS Proceedings Volume AD (2005), 125 - 138.

[17] É. Fusy, D. Poulalhon, and G. Schaeffer, Dissections and trees, with applications to optimal mesh encoding and random sampling, in the Proceedings of the Symposium on Discrete Algorithms (SODA'05), 2005, 690 - 699.

[18] S. Gerke and C. McDiarmid, On the number of edges in random planar graphs, Combinatorics, Probability and Computing, 13 (2004), 165-183.

[19] S. Gerke, C. McDiarmid, A. Steger, and A. Weißl, Random planar graphs with $n$ nodes and a fixed number of edges, In Proceedings of the Sixteenth Annual ACM-SIAM Symposium on Discrete Algorithms (SODA05), 2005, 999 - 1007.

[20] O. Giménez and M. Noy, Asymptotic enumeration and limit laws of planar graphs, available online at http://arxiv.org/abs/math/0501269.

[21] F. Harary and E. M. Palmer, Graphical enumeration, Academic Press, New York (1973).

[22] G. Labelle, C. Lamathe, and P. Leroux, Labelled and unlabelled enumeration of $k$-gonal 2-trees, Journal of Combinatorial Theory, Series A, 106 (2004), 193 - 219. 
[23] V. A. Liskovets, A census of non-isomorphic planar maps, Colloq. Math. Soc. Janos Bolyai, 25 (1981), $479-494$.

[24] C. McDiarmid, A. Steger, and D. Welsh, Random planar graphs, Journal of Combinatorial Theory, Series B, 93 (2005), 187 - 205.

[25] R. C. Mullin and P. J. Schellenberg, The enumeration of c-nets via quadrangulations, Journal of Combinatorial Theory, 4 (1968), 259 - 276.

[26] R.Z. Norman, On the number of linear graphs with given blocks, Doctoral dissertation, University of Michigan, 1954.

$[27]$ D. Osthus, H. J. Prömel, and A. Taraz, On random planar graphs, the number of planar graphs and their triangulations, J. of Combinatorial Theory, Ser. B, 88 (2003), 119 - 134.

[28] R. Otter, The number of trees, Annals of Mathematics, 49 (1948), 583 - 599.

[29] J. M. Plotkin and J. W. Rosenthal, How to obtain a singular expansion of a sequence from an analytic identity satisfied by its generating function, Journal of the Australian Mathematical Society, Series A, Vol. 56, No. 1 (1994), 131 - 143.

[30] G. Pólya, Kombinatorische Anzahlbestimmungen für Gruppen, Graphen und chemische Verbindungen, Acta Mathematica, 68 (1937), 145 - 254.

[31] R. C. Read, On general dissections of a polygon, Aequationes Mathematicae 18, University of Waterloo (1978), $370-88$.

[32] R. W. Robinson, Enumeration of non-separable graphs, Journal of Combinatorial Theory, 9 (1970), $327-356$.

[33] G. Schaeffer, Random sampling of large planar maps and convex polyhedra, in the Proceedings of the 31st annual ACM Symposium on Theory of Computing (STOC'99), 1999, $760-769$.

[34] N. J. A. Sloane, The On-Line Encyclopedia of Integer Sequences, http://www.research. att.com $/ \sim n j$ as $/$ sequences.

[35] W. T. Tutte, A census of planar maps, Canadian J. of Mathematics, 15 (1963), 249 - 271.

[36] Y. Tomii, Gewurzelte Unbeschriftete Outerplanare Graphen, Studienarbeit, HumboldtUniversität zu Berlin, 2005.

[37] S. Vigerske, Asymptotic enumeration of unlabelled outerplanar graphs, Diploma thesis, Humboldt University Berlin, 2005, available online at http://www.informatik. hu-berlin.de/Forschung_Lehre/algorithmen/en/forschung/planar/vigerske.html.

[38] T. Walsh, Counting unlabelled three-connected and homeomorphically irreducible two-connected graphs, Journal of Combinatorial Theory, Series B, 32 (1982), 12 - 32.

[39] T. Walsh and V. A. Liskovets, Ten steps to counting planar graphs, in the Proceedings of Eighteenth Southeastern International Conference on Combinatoris, Graph Theory, and Computing, Congr. Numer. (1987), $269-277$.

[40] H. Whitney, Congruent graphs and the connectivity of graphs, American Journal of Mathematics, 54 (1932), 150 - 168. 